\title{
Interactive Real-Time Control System for The Artificial Hand
}

\author{
Hanadi Abbas Jaber*1, Mofeed Turky Rashid ${ }^{2}$, Luigi Fortuna ${ }^{3}$ \\ ${ }^{1}$ Computer Engineering Department, University of Basrah, Basrah, Iraq \\ ${ }^{2}$ Electrical Engineering Department, University of Basrah, Basrah, Iraq \\ ${ }^{3}$ Department of Electrical, Electronic, Information Engineering, University of Catania, Catania, Italy
}

\begin{abstract}
Correspondence
*Mofeed Turky Rashid

Electrical Engineering Department,

University of Basrah, Basrah, Iraq

Email: mofid76@gmail.com
\end{abstract}

\begin{abstract}
In recent years, the number of researches in the field of artificial limbs has increased significantly in order to improve the performance of the use of these limbs by amputees. During this period, High-Density surface Electromyography (HD-sEMG) signals have been employed for hand gesture identification, in which the performance of the classification process can be improved by using robust spatial features extracted from HD-sEMG signals. In this paper, several algorithms of spatial feature extraction have been proposed to increase the accuracy of the SVM classifier, while the histogram oriented gradient (HOG) has been used to achieve this mission. So, several feature sets have been extracted from HD-sEMG signals such as; features extracted based on $\mathrm{HOG}$ denoted by $(\mathrm{H})$; features have been generated by combine intensity feature with $\mathrm{H}$ features denoted as (HI); features have been generated by combine average intensity with H features denoted as (AIH). The proposed system has been simulated by MATLAB to calculate the accuracy of the classification process, in addition, the proposed system is practically validated in order to show the ability to use this system by amputees. The results show the high accuracy of the classifier in real-time which leads to an increase in the possibility of using this system as an artificial hand.
\end{abstract}

KEYWORDS: HD-sEMG, SVM classifier, Spatial features extraction, HOG approach, Artificial Hand.

\section{INTRODUCTION}

The concept of the prosthesis interaction with the amputees is depending on the electromyography (EMG) signals produced by muscle tissue, which is sensed by electrodes. The local features extracted from EMG signals are used to producing the commands needed to perform prosthetic functions. These prostheses increase the abilities of amputees and patients with physical damage or cognitive functions as a result of disease, injury, and aging [1].

There are two categories of myoelectric controls which are pattern recognition approach and direct control system (conventional). In the direct control, each pair muscle in the opposite site is control the motion of the prostheses. In the case of the artificial limb has a lot of joints movement, a problem will appear due to the existence of a more degree of freedom (DOF) than the control signal obtainable from the muscles of an amputee. This problem may overcome by switching control, which the patient should switch between all available joints. However, switching control is impractical due to a long-time is needed to achieve a complex task [2]. Moreover, the region of muscle activity at which EMG is recorded and measured near-surface of the skin may involve the sharing of more than one muscle that causes the EMG cross-talk. The limitations of both direct and switching control systems led to the use of a pattern recognition approach for myoelectric control, which increases the number of DOF that can be intuitively controlled [3-5].

In the last decades, surface electromyography (sEMG) signals are widely used for pattern recognition, which problems of the signal amplitude value, bad sensing due to fatigue, and abnormal distribution of sEMG on the muscles have not been taken seriously during the most studies of the recognition tasks. The high performance of the pattern recognition process can be achieved by solving the problems of these issues. In recent studies, High-density surface electromyography (HD-sEMG) has been introduced a myoelectric control system, which is a two-dimensional array of electrodes that can give a high performance of recognition by increasing the density of electrodes that cover the most of muscle area [6-11].

Recently, Pattern recognition is the most efficient method for identifying the upper limb gestures from sEMG signals. There are many classifiers that used for pattern 
recognition with a high performance like deep learning approach, artificial neural network (ANN), support vector machine (SVM), linear discriminant analysis (LDA) [12]. Stango [13] uses HD-EMG signals for controlling upper limb prostheses, in which spatial features extracted from the HD-EMG map making the performance of classifier robust to electrode shift but this system has not been solved the problem of the sensitivity reduction due to fatigue or long term of classification. Rojas [14] employs three electrodes array for Biceps, Triceps, and forearm to identify 12 gestures corresponding to four motion types with three effort levels, which spatial features are extracted by mean shift channel algorithm combined with intensity features obtained from five segmented maps corresponds to muscles. However, this system is complex and costly.

Geng et al. $[15,16]$ have been used a 2 dimentional array of electrodes with 128 channels organized as a grid of $8 \times 16$ matrix. Geng deals with the instantaneous recording of HD-EMG as an image, in which a deep convolution network has been implemented for gesture recognition that classifies instantaneous sEMG images, in this system huge data needs for training. In [17], researchers presented a recognition approach based on two categories: wireless HD-EMG recognition system and brain-inspired HD computing in order to achieve high recognition performance, which the proposed system performs. In Rubana [18], several classifiers have been used to analyze the EMG signal, also the comparison is performed between these methods in terms of performance which the high accuracy evaluated by the Extreme Learning Machine (ELM) algorithm, in this system, many problems appear such as complexity, cost, in addition to synchronization problems.

The researchers found that the features of EMG signals are an important factor for identifications and have more impact than classifiers [19]. So, it is best to select simple and fast classifiers for identification like LDA or SVM, which commonly used especially in pattern recognition approaches [20,21]. Furthermore, pattern recognition approaches observed by different studies like subjects of neuromuscular diseases [22] and in fields of the myoelectric control system for prostheses [23-26]. Features that extract from EMG signals can be in time, frequency/scale, and time-frequency/scale domain $[12,20,27]$. Time is the favorite domain due to the simplicity of computation with good performance [20] and can be supported by other features in order to enhance performance [19]. Physiological like muscle stress or non-physiological as skin impedance is affected on the EMG signals in terms of strength and shape, so they are an impact factor on the neuromuscular control [28]. An LDA classifier is proposed by Liu et al. [29], in which the training is performed during different days and then combined the results in order to achieve adaptation for the change in the features.

In this paper, the spatial features are proposed for gesture recognition based on three scenarios; the first one is $\mathrm{H}$ features that corresponds to the histogram oriented gradient (HOG) algorithm while the other method HI features that is related to the combination of $\mathrm{H}$ features and intensity feature that evaluated from the intensity of HD-sEMG map. AIH features related to the same combination of $\mathrm{H}$ features and intensity features as the intensity of the segmented HD-sEMG map. These features are extracted from HD-sEMG signals of eight hand movements of prostheses. The gesture recognized using SVM classifier based features sets. The control system has been designed by a microcontroller in order to control the artificial hand based on classification decisions. Two types of experiments have been achieved in order to validate the proposed system. The first type is the proposed system has been simulated in real-time in order to evaluate the accuracy of classification, while the second type is practical experiments in order to validate the proposed system. The results of the experiments proved that the SVM classification performance is improved by using the proposed features, while the proposed system practically success.

The rest of the paper is organized as follows: in section 2 , interactive control system has been described and study the required tools for this task. In section 3, the simulation of SVM classifier by MATLAB has been performed in order to test the accuracy of using modified HD-sEMG features extraction algorithms. The final section contains the conclusions about the results.

\section{INTERACTIVE CONTROL SYSTEM}

There are many issues emulate the ability of amputees to use artificial limbs such as motion flexibility of hand mechanical structure, decision-making accuracy based on EMG signals, and how much the control system is interactive with an amputee. The complete control system has been drawn in Fig. 1, which the HD-sEMG signals have been sensed by a two dimensional array of electrodes and then sends to a PC in order to decision making based on achieving the classification process for each hand gesture. The gestures identification process is achieved by extracting robust spatial features from HD-sEMG signals that will be employed for the SVM classifier. Finally, based on the decision made by PC, the hand will perform the gestures, which the hand is interfaced with the PC through the microcontroller-based control system. In the following sections, the description of the proposed design will be presented.

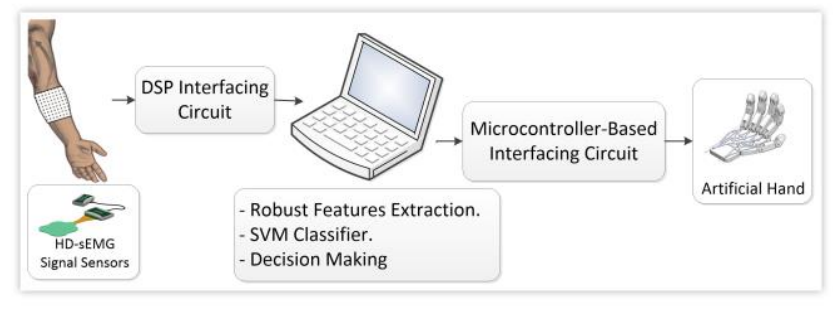

Fig. 1: Interactive Control System.

\section{A. Gestures Identification Approach}

The efficiency of gesture identification is a key factor in controlling prostheses. While the high performance to recognize gestures is subject to many factors [16,30], starting from sensing the HD-sEMG signals of muscles $[13,33]$, and then the method that used for extracting the HD-sEMG signal features [9,13-14], finally, the 
classification method for gesture identification [18, 19,24,27].

\section{1) HD-sEMG Sensing Process}

Electromyography (EMG) signals acquired from electrical activities of muscle by means of surface, needle or implantable electrodes. The electrode may be invasive or non-invasive, the invasive required an interface device between the patient and the robotic prostheses by surgery. Hence, surface EMG electrodes are preferred due to avoiding invasive operation during the recording of signals. The surface electrodes either dry in direct connection with the patient's skin or gelled electrode that uses the chemical material as the interface between skin and electrodes [34]. Recent research showed other types of surface electrodes such as multi-channel electrodes. Multi-channel electrodes use several channels as the linear array or 2D array of surface electrodes known as HD-sEMG electrodes [35,36]. HD-sEMG channels are organized as a two dimensional (2D) grid of closely spaced electrodes covering a muscle area as shown in Fig. 1.

\section{2) HD-sEMG signals database}

In this work, the standard databases of HD-sEMG signals have been used that is commonly used for gesture identification to arrive at a fair comparison with the relevant systems and test the success of the proposed methods with respect to other works. Different types of databases are available such as the CSL-HDEMG database with 192 electrodes arranged in a form of $8 \times 24$ electrodes for upper forearm muscles. It consists of 27 gestures recorded by five subjects. NinaPro is a scientific database has ten spares electrodes on the forearm. In the NinaPro dataset, 27 subjects performed 52 gestures recorded for 10 trials in sub-database1 (DB1) and 40 subjects performed 50 gestures are recorded for 6 trials by sub-database2 (DB2) [15].

The HD-sEMG data obtained from a standard database given at http:// zju_capg.org/myo/data. It consists of 128 channels prepared in matrix form $8 \times 16$ as a $2 \mathrm{D}$ array of closely spaced electrodes. DB-a is considered with 18 subjects. Each subject performed 8 isometric hand gestures. Each gesture repeated for 10 trials [16]. The acquired HD-sEMG data were pre-processed using band-pass filtered at $20-380 \mathrm{~Hz}$ and sampled at $1000 \mathrm{~Hz}$. Each gesture recorded ten trials for each subject. For each trial, the channel was recorded for 1000 samples of instants.

CapgMyo database do not enforce definable contraction force when the subject performed gestures. The opinion of gesture recognition considered a contraction force level is a form of feature [16,36], in which the identification of gestures is easier by imposing the contraction force. This issue produced a limitation in real-world applications, it is difficult to order users to have specific contractions force.

\section{3) $H D$-sEMG features extraction}

HD-sEMG map is the spatial distribution of intensities of the active motor unit over the muscle, in which the sEMG - map is proposed for medical applications, recently, it has been used for gesture recognition. The sEMG map is calculated as

$$
A M_{i, j}=R M S\left(E M G_{i, j}\right)
$$

where $A M_{i, j}$ is activation map of channel $(i, j), E M G_{i, j}$ is the EMG signal at location $(i, j)$ channel. Each pixel in map corresponds to RMS value of a channel at location (i,j).

The HD-sEMG map was calculated for non-overlapping windows (200ms time duration), which is the suggested window length for many studies of pattern recognition based prosthetic control [37]. Then averaged the segmented map as

$$
\begin{gathered}
A S M_{i, j}=\frac{1}{M} \sum_{m=1}^{M} \frac{1}{N} \sum_{n=1}^{N} X_{i j}^{2}(n) \\
A S M_{i, j}=\frac{1}{M} \sum_{m=1}^{M} S M_{i, j}
\end{gathered}
$$

where $\mathrm{ASM}_{\mathrm{i}, \mathrm{j}}$ average segmented map, $\mathrm{X}_{\mathrm{i}, \mathrm{j}}$ is window of EMG signal located at $(\mathrm{i}, \mathrm{j})$ of $2 \mathrm{D}$ arrays, $\mathrm{SM}_{\mathrm{i}, \mathrm{j}}$ is the segmented map located at $(\mathrm{i}, \mathrm{j})$ channel.

$\mathrm{N}$ is total number of samples in each window of sEMG signal, $\mathbf{M}$ is number of non-overlapping windows.

There are different proposed algorithms use to extract features some of them are simple such as RMS value to estimate EMG amplitude or use time domain features (TD) or more complex frequency feature such as Fourier and wavelet domain. New studies display that the spatial distribution of HD-sEMG map improves the recognition of motions. The spatial features relevant to HD-sEMG maps were extracted and used in identification either individual or combined so as to improve their performance $[4,38,39]$. Many studies show that the relation between EMG amplitude and generated force is a non-linear. Therefore, the intensity features were evaluated as a common logarithm of the average intensity of HD-sEMG maps $[25,40]$.

$$
I=\log _{10} \frac{1}{N} \sum_{i, j} A M_{i, j}
$$

where $\mathrm{I}$ is the intensity features, $\mathrm{AM}_{\mathrm{i}, \mathrm{j}}$ is the intensity value of the pixel at location $(i, j)$ of segmented map.

In this study, three sets of feature extraction were presented H, HI, AIH. In these approaches, each channel of HD-sEMG recording signals are divided into several nonoverlapping windows. HD-sEMG maps have been calculated for each window using the RMS value as in (3). The average segmented map has been calculated for each channel to obtain ASM of $8 \times 16$ whereas pixels intensity of this map corresponds to channels location and represents muscle activation map in each channel. The average segmented map (ASM) can be considered as images, therefore, the problem of hand gesture can be reframed as the problem of image classification. The Histogram Oriented Gradient (HOG) algorithm is applied to extract the spatial features of the average HD-sEMG map. HOG is an efficient feature extraction technique, which is widely used in image processing for the purpose of object detection. HOG counts the occurrences of gradient orientation in localized portions of an image [41]. The extracted HOG features from the average HD-sEMG map denoted as $\mathrm{H}$ features, which used for the gesture classification process.

The second proposed feature set is concatenated of intensity feature (I) and $\mathrm{H}$ feature within a single vector of the feature, in which the intensity feature has been calculated 
by equation (4) as a scalar value. This feature set is denoted as HI.

In the third proposed feature, the intensity feature is calculated by averaging the segmented maps per channel to represent a vector of $1 \times 128$ features corresponds to the number of channels of HD-sEMG array. This intensity vector is concatenated with $\mathrm{H}$ features to form $\mathrm{AIH}$ features. The three proposed feature sets have been illustrated as a flowchart in Fig. 2.

\section{4) HD-sEMG Classification Process}

The simple implementation and fast training of the SVM classifier encouraged the use of this classifier for classifying eight hand gestures by finding the best hyperplane that separates multi classes. The linear kernel was used that achieved preferable accuracy than other kernels [1]. Three different SVM classifier has been implemented according to different feature sets extracted from HD-sEMG data

- Classifier based on $\mathrm{H}$ features.

- Classifier based on HI features.

- Classifier based on AIH features.

In order to achieve an honest assessment of the true accuracy of our classifier, 10 fold cross-validation has been used. the performance of the classifier has been evaluated in term of sensitivity $(\mathrm{S})$ and Precision $(\mathrm{P})$ that computed from the confusion matrix as

$$
\left.\begin{array}{l}
S=\frac{T_{P}}{T_{P}+F_{N}} \\
P=\frac{T_{P}}{T_{P}+F_{P}}
\end{array}\right\}
$$

where $T_{P}$ (true positive) is the number of samples that were classified properly to a specific class; $\mathrm{T}_{\mathrm{N}}$ (true negative) is the number of samples that do not pertain to a definite class and were not categorized to that class; $F_{N}$ (false negative) is the number of samples pertaining to a specific class but erroneously classified into another class; $\mathrm{F}_{\mathrm{P}}$ (false positive) is the number of samples not pertaining to a definite class but incorrectly classified into that class [26,42]. Figure 3 shows the systematic representation of SVM classifier based on AIH features.

The classification accuracy (CA) is used for real time evaluation of individual stream. It is calculated as

$$
\text { CA } \%=\frac{\text { correctly predicted samples }}{\text { total test } \text { samples }} \times 100
$$

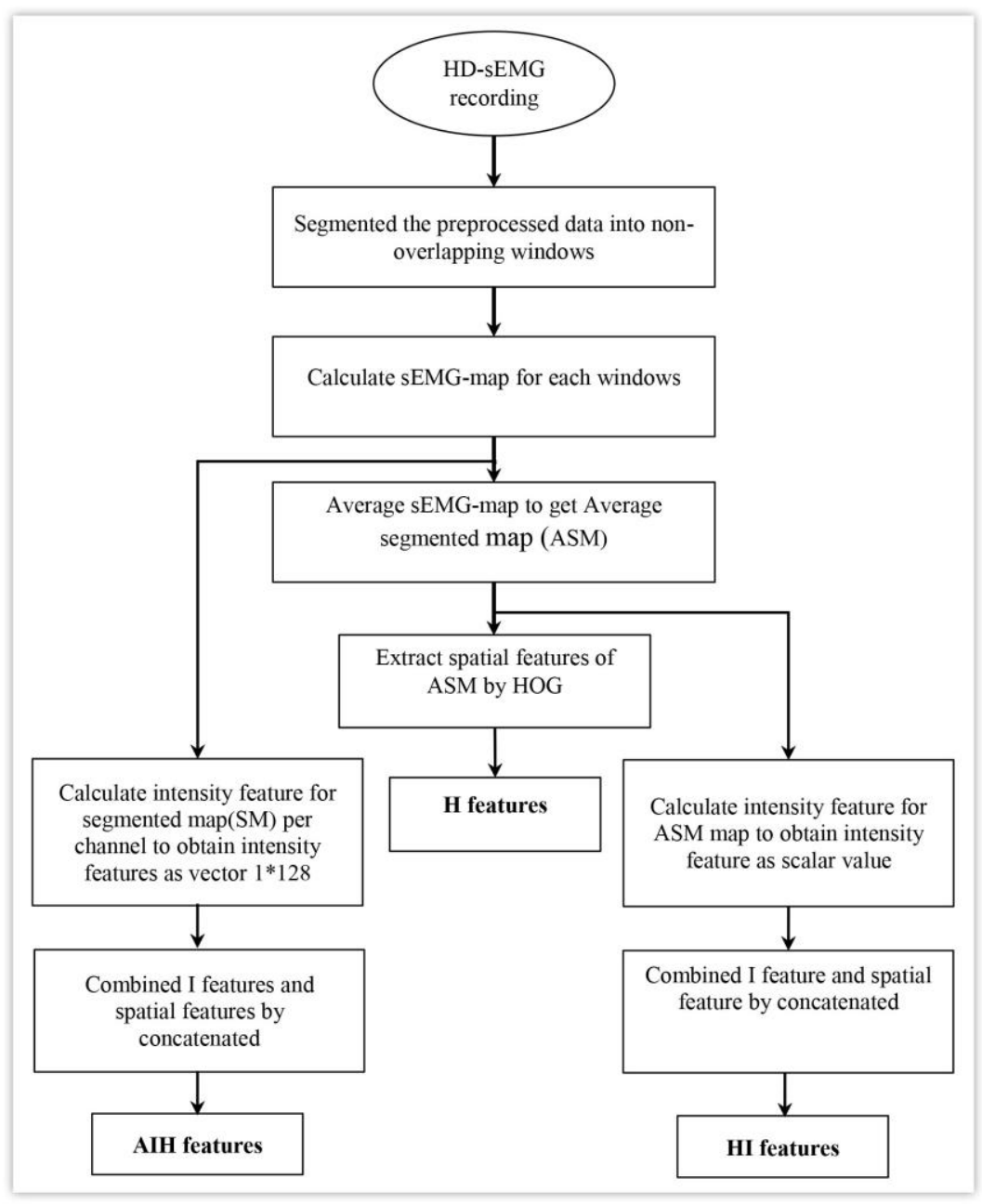

Fig. 2: Features extraction flowchart. 


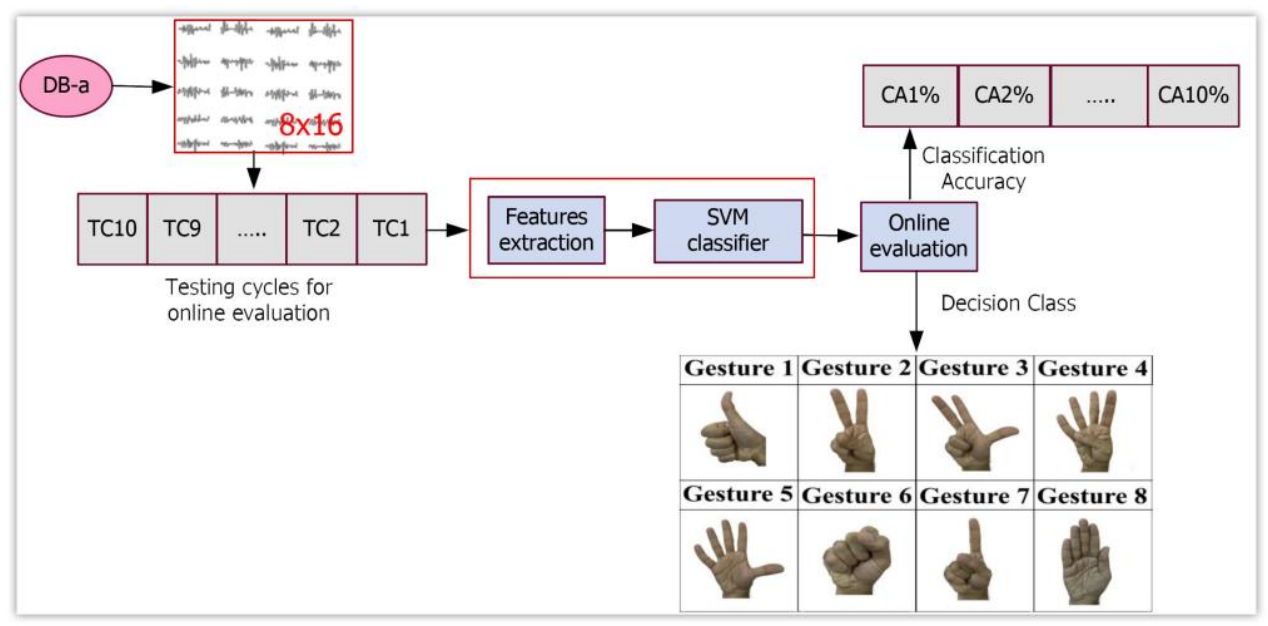

Fig. 3: The systematic representation of SVM classifier based on AIH features.

\section{B. Implementation of Myoelectric Control System}

In this section, the myoelectric control system has been implemented, which is shown in Fig. 4. As mentioned in previous sections the HD-sEMG system is used for sensing a 2D array of EMG signals. In this paper, the HD-sEMG signals from a dataset have to be employed, and then spatial features are extracted. While these signals will be used for the real-time classification that achieved in Laptop by MATLAB software. The classification process is followed by decision making in order to achieve gestures, while the decision will be sent through a PC interfacing circuit to the artificial hand control system. Microcontroller type ATMEGA 2560 has been used for the main control unit, which is receiving the decision from PC. According to these decisions, instruction making will be generated in order to drive the artificial hand. The Artificial hand has a simple design, which made from metal material with five fingers for each finger there are 3-DOF of three revolute joints.

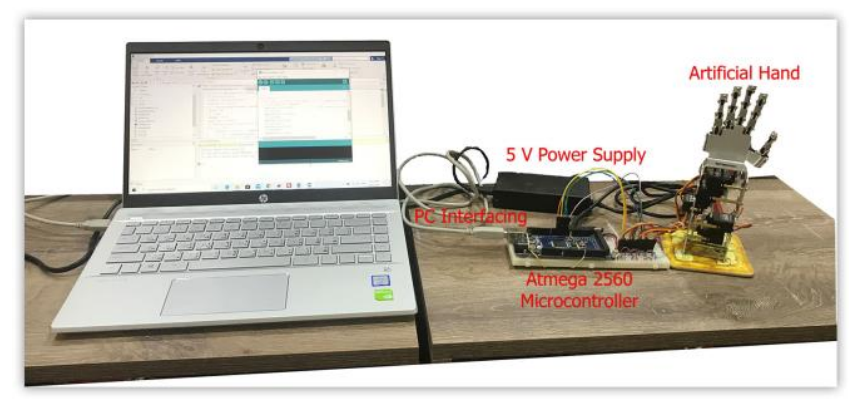

Fig. 4: Implementation of myoelectric Control System.

\section{EXPERIMENTS AND RESULTS}

Several experiments have been implemented in order to validate the proposed system. These experiments start to simulating the HD-sEMG signal classification to identify the hand gesture, which accuracy, precision, and sensitivity have been evaluated for performance measurement. As mentioned in the previous section, the HD-sEMG electrodes have been organized as a 2D array in 8 rows and 16 columns. The identification is tested on the first eight able-bodied subjects of database DB-a for the classification of eight hand gestures. The classifier was trained using five trials and used the remaining five trails for testing. (50\% training set, 50\% test set). The offline evaluation confirms the powerful of the proposed feature sets. The averaged precision and sensitivity of all gestures are displayed in Table I. There is a significant difference of precision and sensitivity based on AIH features compared with $\mathrm{H}$, $\mathrm{HI}$ features (i.e. $\mathrm{P}_{\mathrm{AIH}}=97.9, \mathrm{~S}_{\mathrm{AIH}}=97.5$ corresponds to $\left.\mathrm{P}_{\mathrm{H}}=93.8, \mathrm{P}_{\mathrm{HI}}=94.4, \mathrm{~S}_{\mathrm{H}}=91.5, \mathrm{~S}_{\mathrm{HI}}=92.5\right)$. Moreover, AIH features achieved lower standard deviation (approximately $1 \%, 5 \%$, and $4.78 \%$ ) than $\mathrm{H}, \mathrm{HI}$ features $(2.76 \%, 11.2 \%, 14.87 \%$ and $2.68 \%, 11.35 \%, 13.56 \%$ respectively). While, slight difference is noticed between the performances of SVM classifier based on $\mathrm{H}$ feature and $\mathrm{HI}$ features.

\section{TABLE I}

The Average Accuracy, Precision, AND Sensitivity AVERAGEd BETWEEN EIGHT SUBJECTS, EIGHT GESTURES AND PRESENTED IN TERM OF MEAN AND STANDARD DEVIATION.

\begin{tabular}{|l|l|l|}
\hline \multicolumn{1}{|c|}{ Features sets } & Average P & \multicolumn{1}{c|}{ Average S \% } \\
\hline H features & $93.8 \pm 11.2$ & $91.5 \pm 14.87$ \\
\hline HI features & $94.4 \pm 11.35$ & $92.5 \pm 13.56$ \\
\hline AIH features & $97.9 \pm 5$ & $97.5 \pm 4.78$ \\
\hline
\end{tabular}

In order to implement the real time evaluation of three feature sets $(\mathrm{H}, \mathrm{HI}, \mathrm{AIH})$, the DB-a is divides into ten streams such that five streams are used for training the classifier in offline while the rest used for on line evaluation. 
The classification accuracy is evaluated for each stream in real time individually. The classification accuracy of five streams based on three feature sets $(\mathrm{H}, \mathrm{HI}, \mathrm{AIH})$ are shown in Fig. 5.

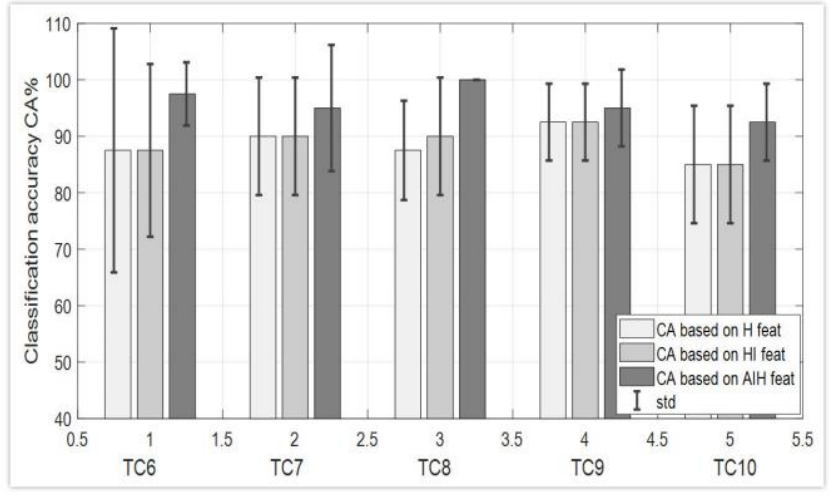

Fig. 5: The Classification Accuracy of five streams (TC6, TC7, TC8, TC9, TC10) based on three feature sets average between five subjects in term of mean and standard deviation.
It is obvious that the classification accuracy of three SVM classifiers based on three feature sets achieved good results for all online five streams always above $85 \%$. Moreover, AIH considerably outperform all of the compared features and its performance is higher than others feature sets. This is explicit from the average accuracy that is always above $92.5 \%$. Further, AIH features have been satisfied relative lower standard deviation than other feature sets as can be seen in TC6, TC8, TC10. This reveals the robustness of AIH features to predict the gestures for five batches online than other features. The superiority of AIH features due to the spatial intensity features of individual channels that are repeated between trials that belong to the same gestures.

Other experiment is illustrated in this work that achieved practical implemented of a hand prosthesis. Classification of six gestures are employed for arbitrary subject (e.g. subject 4 is chosen). The confusion matrix is described for four streams of subject 4 based on three feature sets (H, HI, AIH features) as shown by Fig. 6, Fig. 7, Fig. 8. Respectively.

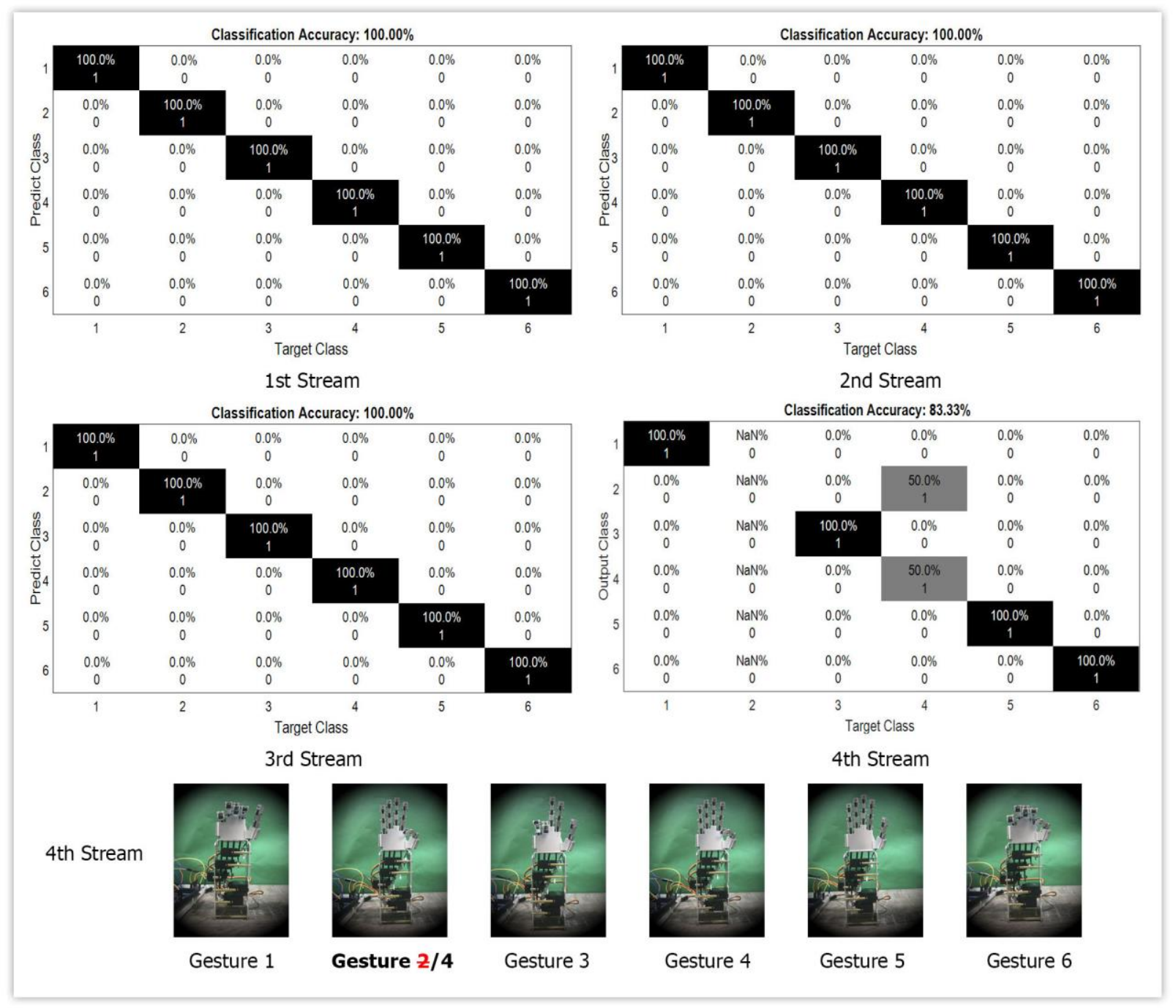

Fig. 6: Confusion matrix for four online streams evaluation respectively based on $\mathrm{H}$ features. 


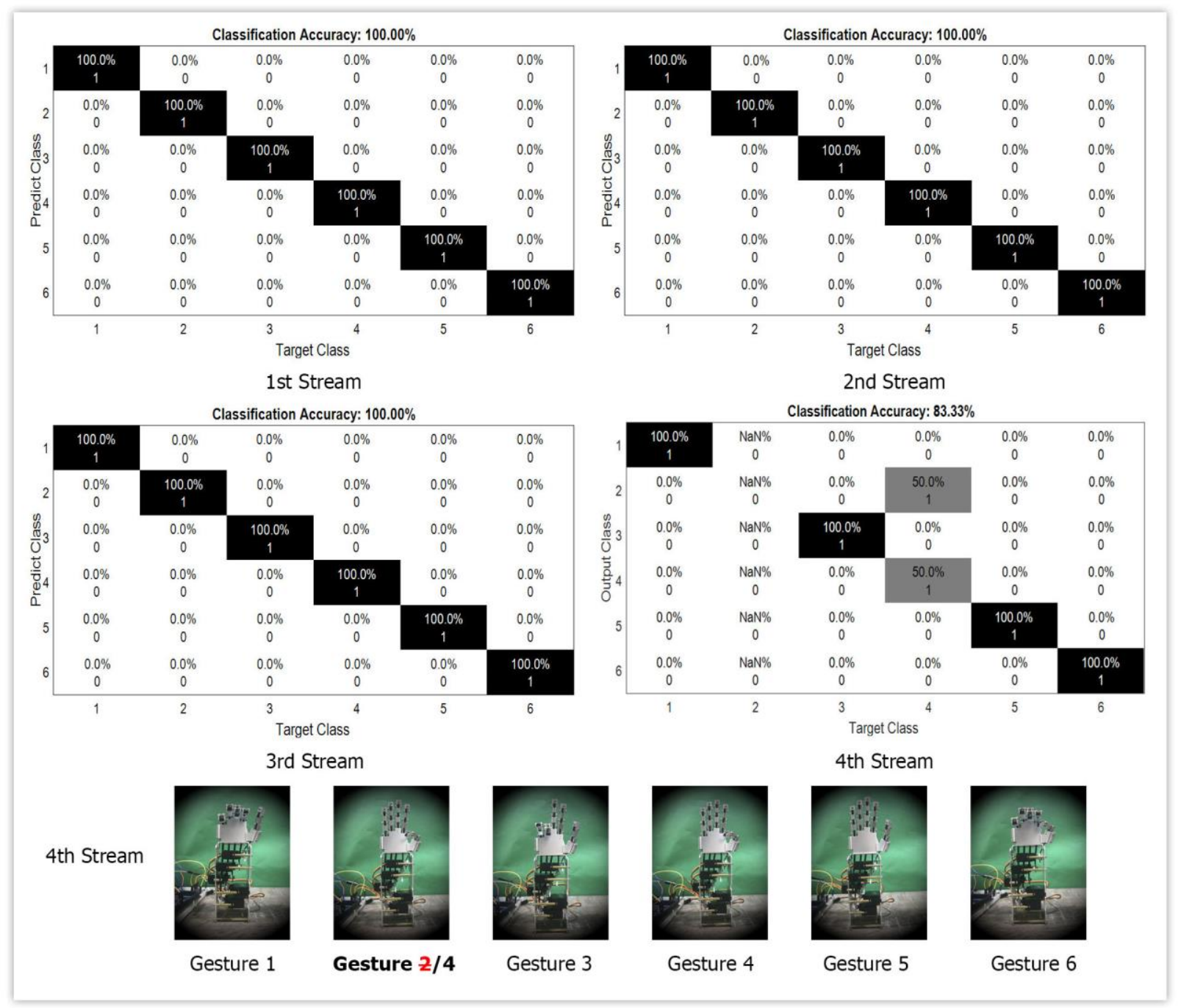

Fig. 7: Confusion matrix for four online streams evaluation respectively based on HI features.

As can be noticed by Fig. 6 all gestures are discriminated correctly except gesture2. There is a misclassification in 4th stream such that gesture 2 cannot be recognized, it is predicted as gesture 4 . The classification accuracy of stream 4 is corresponds to classify 5 gestures correctly versus one misclassification. Hence, the classification accuracy is $83.33 \%$. The same online evaluation is satisfied for HI features as shown by Fig. 7. While online evaluation of $\mathrm{AIH}$ features can recognize all gestures for all four streams for that subject with $100 \%$ classification accuracy.

In order to show the classification accuracy of each subjects, the classification accuracy $(\mathrm{CA} \%)$ is calculated for each subject averaged between testing stream. The performance of online classifier based on AIH features for five subjects with their average accuracy are shown in Fig. 9. It is clear that the performance significantly changes between subjects. inter-subject variation due to human anatomical such that the motor units in the muscle tissue is different between subjects. However, the average classification accuracy produced an acceptable performance above $92.5 \%$. This gives an indication that the choice of feature set is more important than the choice of the classifier, and has a significant effect on the performance of classifier. As a summary Table II include the classification accuracy of the related work. Indeed, the comparison of the related work isn't fair due to several factors such as different databases used, increased number of task identification, number of subjects that entrant in the experiment. However, this table display the scope of the classification accuracy with respect to features, classifier and technique for recording EMG signals used. Stango, Phinyomark [13,43] used the same classifier of our work (SVM) to classify 9,7 movements with average accuracy $95 \%, 88.6 \%$ respectively using two different feature sets.

Geng in [16] verified the maximum accuracy of 99.5 by using the powerful of CNN deep learning method based on instantaneous image of 128 channels. In our study, SVM classifier based AIH features achieved high classification accuracy reach to $97.5 \%$. This gives an indication that the choice of feature set has a significant impact on the identification process. Further the spatial features have been influenced the classification performance. 


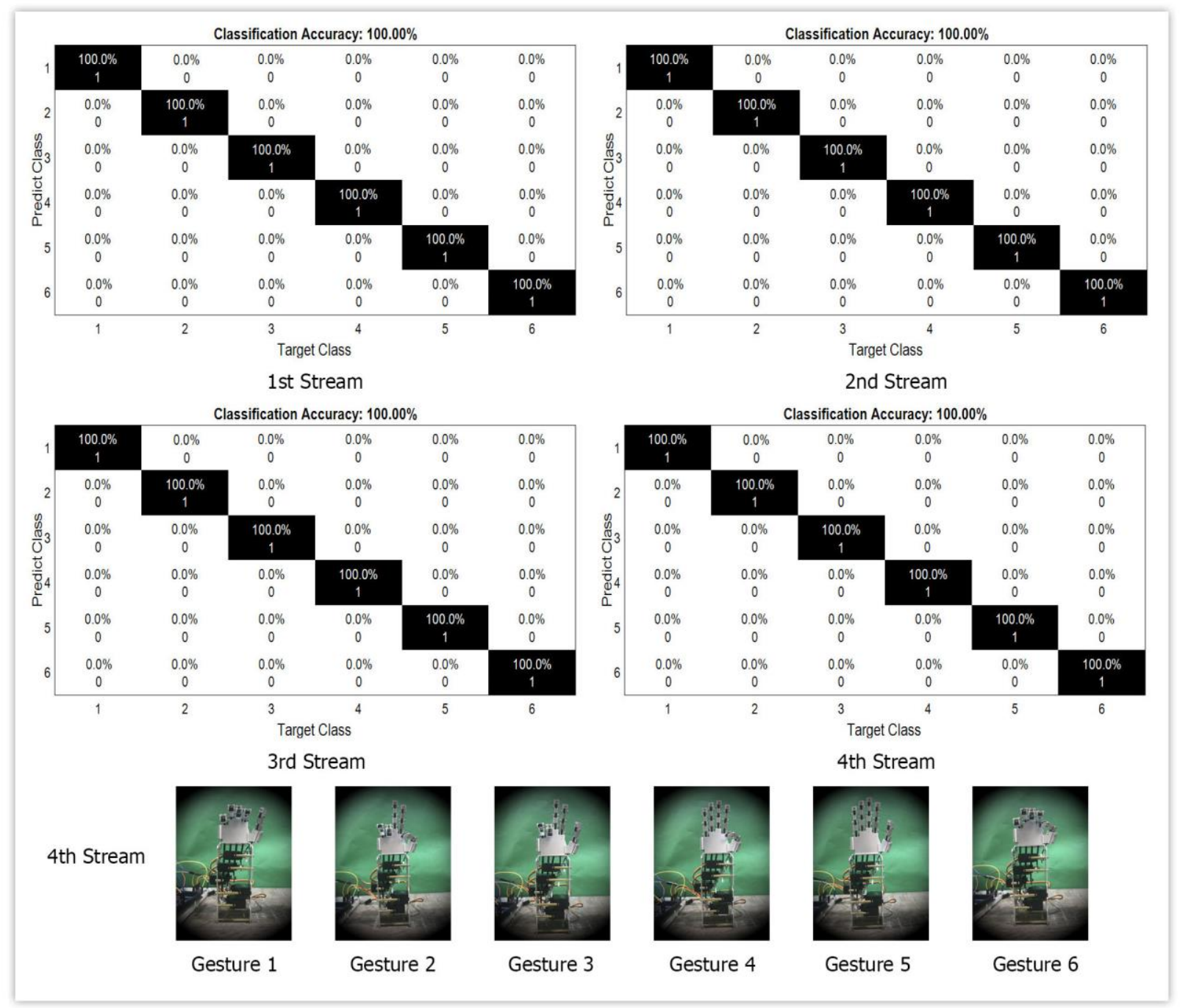

Fig. 8: Confusion matrix for four online streams evaluation respectively based on AIH features

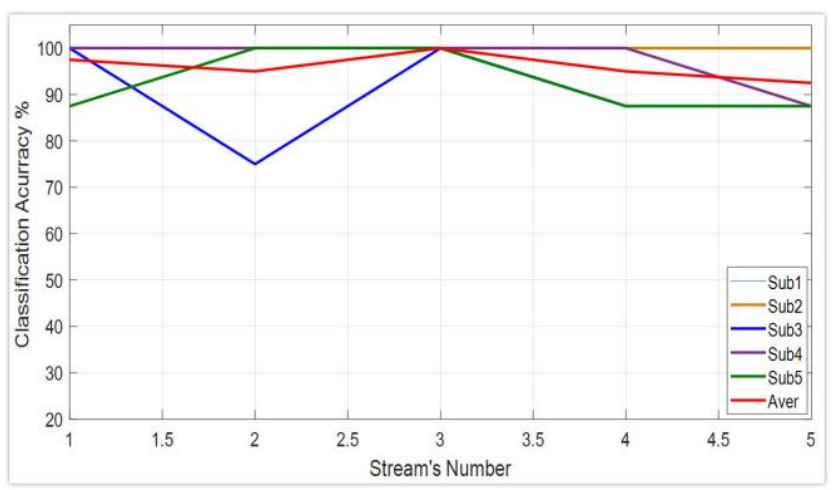

Fig. 9: the classification accuracy based on AIH features for five subjects with dsdwtheir average

\section{CONCLUSIONS}

Five subjects performed eight gestures. HD-sEMG data has been obtained from the standard database CapgMyo database DB-a. It consists of 128 channels configured as $8 \times 16$ arrays, each channel recorded for 1000 samples instants. Each channel is divided into 5 frames of $200 \mathrm{~ms}$ non-overlapping windows. HD-EMG map is calculated for each segmented window by RMS value then averaged segmented maps to obtain the average segmented map. Different feature sets have been extracted $\mathrm{H}$ feature corresponds to spatial features extracted from the average segmented map that considered as an image of $8 \times 16$ by the HOG algorithm. HI features obtained by combining $\mathrm{H}$ features and intensity features. The last evaluated as intensity feature of the average segmented map. AIH features related to combined $\mathrm{H}$ features and vector of intensity features that calculated for segmented maps per channel. The gesture recognized using SVM classifier based features sets. AIH features have been achieved higher performance (i.e. $\mathrm{P}=97.9 \pm 5, \mathrm{~S}=97.5 \pm 4.87$ ) with lower standard deviation. So, the spatial and intensity features considerably improved the classification of motion intents. 
TABLE II

COMPARISON BETWEEN THE PERFoRMANCE OF GESTURES IDENTIFICATION BASED ON AIH, FEATURES AND PERFORMANCE OF GESTURES IDENTIFICATION THAT ACHIEVED BY OTHER RESEARCHERS.

\begin{tabular}{|c|c|c|c|c|c|c|}
\hline Reference & $\begin{array}{c}\text { Classification } \\
\text { accuracy }\end{array}$ & $\begin{array}{c}\text { Nu mber of } \\
\text { movements }\end{array}$ & $\begin{array}{c}\text { Number of } \\
\text { subjects }\end{array}$ & $\begin{array}{c}\text { Type of Features } \\
\text { Channels } \\
\text { type }\end{array}$ & I+CG & $\begin{array}{c}\text { 120 for 2-array (Biceps and Triceps) } \\
114 \text { for forearm array }\end{array}$ \\
\hline Rojas [25] & $98.7 \%$ & 4 & 9 & LDA & 192 \\
\hline Geng [16] & $99.5 \%$ & 8 & 18 & $\begin{array}{c}\text { Instantaneous values of EMG } \\
\text { signal as feature vectors }\end{array}$ & SVM \\
\hline Ali [17] & $97.9 \%$ & 5 & 3 & Spatial HD vector & Deep learning \\
\hline Phinyomark [43] & $88.6 \%$ & 7 & 11 & TD4 & Myo armband \\
\hline Our work & $\mathbf{9 7 . 5 \%}$ & $\mathbf{8}$ & $\mathbf{8}$ & AIH & HD classifier \\
\hline
\end{tabular}

\section{CONFLICT OF INTEREST}

The authors have no conflict of relevant interest to this article.

\section{REFERENCES}

[1] P. M. Pilarski, M. R. Dawson and R. S. Sutton, "Adaptive artificial limbs, A real time approach to prediction and anticipation", IEEE Robotic and Automation Magazine, IEEE Robotics \& Automation Magazine, Vol. 20, Issue 1, pp. 53-64, March 2013.

[2] Hanadi A. Jaber, Mofeed T. Rashid and L Fortuna, "Using the Robust High Density-surface Electromyography Features for Real-Time Hand Gestures Classification", IOP Conference Series: Materials Science and Engineering, 1 Feb. 2020.

[3] D. Farina, N. Jiang, H. Rehbaum, "The extraction of neural information from surface EMG for the control of upper limb prosthesis Emerging and challenging", IEEE Trans. Neural Syst. Rehabil. Eng., Vol. 22, No. 4, pp. 797-808, July 2014.

[4] H. A. Jaber, M. T. Rashid, L. Fortuna, "Robust Hand Gesture Identification Using Envelope of HD-sEMG Signal", ACM international conference proceeding series, 2019.

[5] N. Meselmani, M. Khrayzat, K. Chahine, "pattern recognition of EMG signals: towards adaptive control of Robotic Arm", IEEE International Multidisciplinary Conf. on Eng. Tech., 2016.

[6] M. Rojas-Martinez, M. A. Mananas, J. F. Alonso, R. Merletti, "Identification of isometric contractions based on High Density EMG maps", Journal of Electromyography and Kinesiology, Vol. 23, pp. 33-42, 2013.

[7] H. A. Jaber, M.T. Rashid, "HD-sEMG Gestures Recognition by SVM Classifier for Controlling Prosthesis", Iraqi Journal of Computers, Communications, Control and System Engineering(IJCCCE), Vol. 19, No. 1, 2019.

[8] Phinyomark A., Quaine F., Charbonnier S., Serviere C., Tarpin-Bernard F., Laurillau Y., "EMG Feature Evaluation for Improving Myoelectric Pattern Recognition Robustness", Expert Syst. Appl., Vol. 40, pp. 4832-4840, 2013.
[9] Tkach D., Huang H., Kuiken T. A., "Study of Stability of Time-Domain Features for Electromyographic Pattern Recognition", J. NeuroEng. Rehabilit, Vol. 7, No. 21, 2010.

[10] Phinyomark A., Quaine F., Charbonnier S., Serviere C., Tarpin-Bernard F., Laurillau Y., "A Feasibility Study on the Use of Anthropometric Variables to Make Muscle-Computer Interface More Practical", Eng. Appl. Artif. Intell., Vol. 26, pp. 1681-1688, 2013.

[11] Khushaba R. N., Al-Timemy A., Kodagoda S., Nazarpour K., "Combined Influence of Forearm Orientation and Muscular Contraction on EMG Pattern Recognition”, Expert Syst. Appl., Vol. 61, pp. 154-161, 2016.

[12] Oskoei M. A., Hu H., "Myoelectric control systems-A survey", Biomed. Signal Process. Control, Vol. 2, pp. 275-294, 2007.

[13] A. Stango, F. Negro, D. Farina, " Spatial correlation of High Density EMG signals provided features robust to electrode number shift in pattern recognition for Myocontrol", IEEE Trans. Neural Syst. Rehabil. Eng., Vol. 23, No. 2, pp.189-198, March 2015.

[14] M. Jordanic, M. Rojas, M. A. Mananas, J. F. Alonso, Sensors, V17, 2017, "A novel spatial features for the identification of motor tasks using HD-EMG".

[15] Geng W., Du Y., Jin W., Wei W., Hu Y., Li J., "Gesture recognition by instantaneous surface EMG images", Sci. Rep., Vol.6, 2016.

[16]Du Y., Jin W., Wei W., Hu Y., Geng W., "Surface EMG-based inter-session gesture recognition enhanced by deep domain adaptation", Sensors, Vol. 17, 2017.

[17] Ali Moin, Andy Zhou, Abbas Rahimi et al., "An EMG Gesture Recognition System with Flexible High-Density Sensors and Brain-Inspired High-Dimensional Classifier", IEEE International Symposium on Circuits and Systems (ISCAS), 2018.

[18] Rubana H. Chowdhury, Mamun B. I. Reaz, Mohd Alauddin Bin Mohd Ali, Ashrif A. A. Bakar, Kalaivani Chellappan and Tae G. Chang, "Surface Electromyography Signal Processing and Classification Techniques", Sensors, Vol. 13, pp.12431-12466, 2013.

[19] Hargrove L. J., Englehart K., Hudgins B., "A comparison of surface and intramuscular myoelectric signal classification", IEEE Trans. Biomed. Eng., Vol. 54, pp. 847-853, 2007. 
[20] Hakonen M., Piitulainen H., Visala A., "Current state of digital signal processing in myoelectric interfaces and related applications", Biomed. Signal Process. Control, Vol.18, pp. 334-359, 2015.

[21]Huang H., Zhou P., Li G., Kuiken T., "Spatial filtering improves EMG classification accuracy following targeted muscle reinnervation", Ann. Biomed. Eng., Vol. 37, pp. 1849-1857, 2009.

[22] Celadon N., Dosen S., Binder I., Ariano P., Farina D., "Proportional estimation of finger movements from high-density surface electromyography", J. Neuroeng. Rehabil., Vol. 13, No. 73, 2016.

[23] Ameri A., Englehart K. B., Parker P. A., “A comparison between force and position control strategies in myoelectric prostheses", In Proceedings of the 2012 Annual International Conference of the IEEE on Engineering in Medicine and Biology Society (EMBC), San Diego, CA, USA, pp. 1342-1345, 28 August-1 September 2012.

[24]Li Z., Wang B., Yang C., Xie Q., Su C. Y., "Boosting-based EMG patterns classification scheme for robustness enhancement", IEEE J. Biomed. Heal. Inform., Vol. 17, pp. 545-552, 2013.

[25] Jordanic M., Rojas-Martínez M., Mananas M. A., Alonso J. F., "Spatial distribution of HD-EMG improves identification of task and force in patients with incomplete spinal cord injury", J. Neuroeng. Rehabil., Vol. 13, No. 41, 2016.

[26] Jordani'c M., Rojas-Martínez M., Mananas M. A., Alonso J. F., "Prediction of isometric motor tasks and effort levels based on high-density EMG in patients with incomplete spinal cord injury", J. Neural Eng., Vol. 13, 2016.

[27] Nazmi N., Abdul Rahman M., Yamamoto S. I., Ahmad S., Zamzuri H., Mazlan S., "A Review of Classification Techniques of EMG Signals during Isotonic and Isometric Contractions", Sensors, Vol. 16, 2016.

[28] Vidovic M. M. C., Hwang H. J., Amsuss S., Hahne J. M., Farina D., Muller K. R., "Improving the robustness of myoelectric pattern recognition for upper limb prostheses by covariate shift adaptation", IEEE Trans. Neural Syst. Rehabil. Eng., Vol. 24, pp. 961-970, 2016.

[29] Liu J., Sheng X., Zhang D., Jiang N., Zhu X., “Towards zero retraining for myoelectric control based on common model component analysis", IEEE Trans. Neural Syst. Rehabil. Eng., Vol. 24, pp. 444-454, 2016.

[30] Li Y., Chen X., Zhang X., Zhou P., "Several practical issues toward implementing myoelectric pattern recognition for stroke rehabilitation", Med. Eng. Phys., Vol. 36, 754-760, 2014.

[31]E. Scheme and K. B. Englehart, "Electromyogram pattern recognition for control of powered upper-limb prostheses: State of the art and challenges for clinical use", J. Rehab. Res. Develop., Vol. 48, No. 6, pp. 643-660, 2011.

[32] Naik G. R., Kumar D. K., Palaniswami M., "Surface EMG based hand gesture identification using semi blind ICA: Validation of ICA matrix analysis", Electromyogr Clin Neurophysiol, Vol. 48, pp.169-80, 2008.
[33] Dick F. Stegeman, Bert U. Kleine, Bernd G. Lapatki, Johannes P. Van Dijk, "High-density Surface EMG: Techniques and Applications at a Motor Unit Level", Biocybernetics and Biomedical Engineering, Vol. 32, No. 3, pp. 3-27, 2012.

[34] J. Kilby, K. Prasad , G. Mawston, "Mutli-channel surface electromyography electrodes: A Review", IEEE Sensors Journal, Vol. 16, Issue 14, pp. 5510-5519, 2016.

[35]C. C. Cordova, C. Ramirez, " EMG signal pattern recognition based on feedforward artificial neural network applied to robotic prosthesis Myoelectric control," Future technology conference, 2016.

[36] Phinyomark, A. Phukpattaranont, P. Limsakul, "Feature reduction and selection for EMG signal classification", Expert Systems with Applications, Vol. 39, Issue 8, pp. 7420-7431, 15 June 2012.

[37] L.H. Smith, L.J. Hargrove, B.A. Lock, T.A. Kuiken, "Determining the optimal window length for pattern recognition based myoelectric control: Balancing the competing effects of classification error and controller", IEEE Trans. Neural Syst. Rehabil. Eng., Vol. 19, No. 2 pp. 186-192, Aprilm 2011.

[38] Hakonen M, Piitulainen H, Visala A, "Current state of digital signal processing in myoelectric interfaces and related applications", Biomed Signal Process Control Elsevier Ltd., Vol. 18, pp. 334-59, 2015.

[39]Zhang X, Zhou P., "High-Density Myoelectric Pattern Recognition Toward Improved Stroke Rehabilitation", IEEE Trans Biomed Eng., Vol. 59, No. 6, pp. 1649-57, 2012.

[40] Staudenmann D., Roeleveld K., Stegeman D. F., Dieen J. H., "Methodological aspects of SEMG recordings for force estimation - A tutorial and review", J. Electromyography Kinesiol, Vol. 20, No. 3, pp. 375-87, 2010.

[41] Luo H., Yu X., Liu H., Ding Q., “A method for real-time implementation of HOG feature extraction", Proc. of SPIE, Vol. 8193, 2011.

[42] M. Rojas, M.A. Mananas, J. F. Alonso, "High-Density surface EMG maps from upper -arm and forearm muscles", J. of Nuero Eng. and Rehabil., Vol. 9, No. 85, 2012.

[43] A. Phinyomark, Rami N. Khushaba, and Erik Scheme, "Feature Extraction and Selection for Myoelectric Control Based on Wearable EMG Sensors", sensors MPDI, Vol. 18, 1615, 2018. 